Document downloaded from:

http://hdl.handle.net/10251/65959

This paper must be cited as:

Ocana-Levario, S.; Ayala Cabrera, D.; Izquierdo Sebastián, J.; Pérez García, R. (2015). 3D model evolution of a leak based on GPR image interpretations. Water Science and Technology: Water Supply. 15(6):1312-1319. doi:10.2166/ws.2015.093.

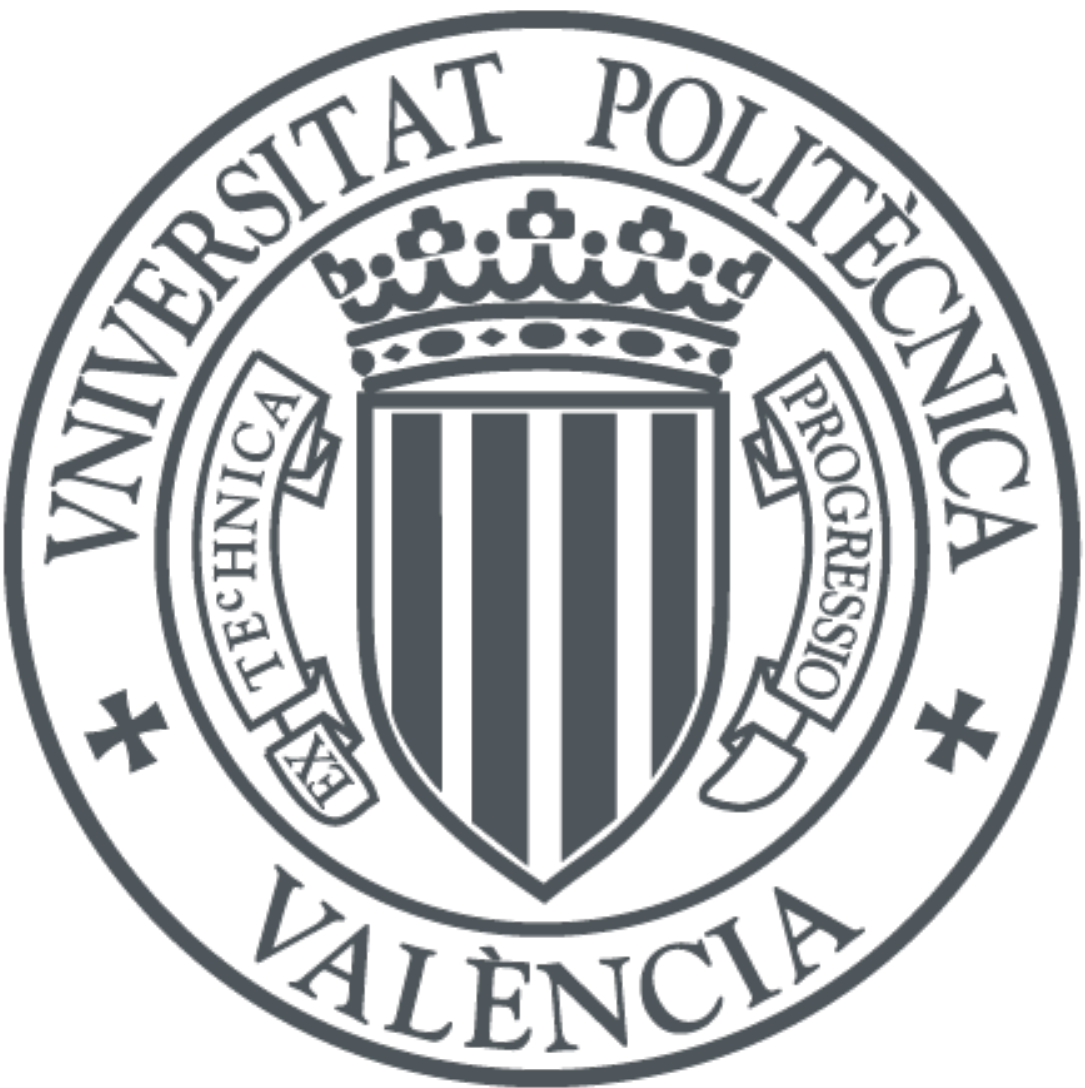

The final publication is available at

http://dx.doi.org/10.2166/ws.2015.093

Copyright IWA Publishing

Additional Information 


\title{
3D model evolution of a leak based on GPR image interpretation
}

\author{
Silvia J. Ocaña-Levario ${ }^{a}$, David Ayala-Cabrera ${ }^{a^{*}}$, Joaquín Izquierdo ${ }^{a}$, Rafael Pérez- \\ García $^{a}$ \\ ${ }^{a}$ FluIng-IMM, Universitat Politècnica de València, Camino de Vera s/n, Valencia, \\ 46022, Spain. \\ *Email: daaycab@upv.es
}

\begin{abstract}
This paper presents some aspects regarding time propagation of underground water leakage in controlled laboratory conditions using a drilled PVC pipe and interpreting ground penetrating radar (GPR) images. GPR pre-processed images are interpreted for an easy identification and extraction of surfaces and volumes of water leakage. Finally, the temporal evolution of a water leak is shown using 3D models based on interpretation of GPR images. Water volumes obtained using this approach can be easily observed by personnel who lack highly specialized training in the analysis of raw data. The results of this study are promising and can help develop techniques to validate non-destructive models for the identification, distribution, and prediction of water leaks in water supply systems using GPR.
\end{abstract}

Keywords: ground penetrating radar, GPR interpretations, leak visualization, water leakage evolution, water supply systems.

\section{INTRODUCTION}

Water leakage detection and control is one of the greatest challenges in the management of water supply systems (WSSs). This paper addresses the problem of leakage in WSSs from the perspective of non-destructive methods.

The most popular non-destructive methods currently used to locate leaks in WSSs are: acoustic methods; infrared thermography; and ground penetrating radar (GPR) (Demirci et al. 2012). GPR has been shown to be an effective non-destructive tool by showing in radargram images the contrast between leaked water and the surrounding area - the contrasts being caused by dielectric differences (Crocco et al. 2010, Charlton et al. 2001). We performed laboratory tests to extract features of water leakage from GPR images. Temporal evolution of water leakage was the initial feature evaluated in this work, supported by leak evolution tracing via 3D modeling.

The main idea was to produce reliable knowledge and robust data that facilitated an understanding of the phenomenon through 3D representation, thus favoring the prediction and/or detection of water leakage in the system. The aim was to increase the approximation abilities of the model, so as to improve operational and maintenance activities and favor decision-making in WSS management. This is possible because GPR image interpretation enables identifiable surfaces (with variable grade difficulty) to be obtained and this then enables objects or phenomena underground to be rebuilt in 
3D models. This process facilitates the successful use of GPR data by personnel who are not highly qualified.

In this paper, water leak propagation from a buried PVC pipe is addressed. This is performed using GPR images obtained under controlled laboratory conditions. Plastic material was selected because of the difficulty experienced in identifying plastic pipes when using GPR (Ayala-Cabrera et al. 2011) and the decreased visibility of surrounding objects caused by plastic materials (Ayala-Cabrera et al. 2013b).

Several authors have addressed leak detection, including Eyuboglu et al. (2003), Hasan (2012), Demirci et al. (2012), Cataldo et al. (2014). These works are promising with respect to GPR usage in WSS leak detection. However, all of these works are based on the location and interpretation of the hyperbolas generated either in raw or preprocessed images. In this sense, we evaluate the viability of applying a pre-processing GPR image methodology that the authors term agent race (Ayala-Cabrera et al. 2013a). With this approach, we aim to eliminate image contrast requirements, as used in AyalaCabrera et al. (2014), and follow the evolution of a water leak from GPR images - so that the contrast can be revealed using the information from events registered in GPR images based on the same data set. If this approach is possible, then the application of GPR in uncontrolled soil conditions and without contrast could be significantly improve images. Other interesting works show support for this line of research: with nondestructive methods for plume dispersion of contaminants (Moradi et al. 2011) in the case of geophysical methods for delineating plumes caused by oil contamination through soil and groundwater. Pollution plume evaluation in an urban environment is also addressed in Vaudelet et al. (2011). Knowledge obtained with the methodology described in this paper may provide basic know-how for other engineering fields in such aspects as fluid dispersion through subsoil, infrastructure risks associated with water leaks, and wet bulb distribution for irrigated areas.

This document has been organized as follows: the first section contains a brief introduction of the tasks performed. The general approach along with the difficulties and benefits is presented in Section 2. The third section presents a case study and survey characteristics. The approach used to interpret and classify the objects obtained by the GPR images is presented in the fourth section. The fifth section gathers the resulting interpretation and classification analysis. The paper ends with conclusions.

\section{OVERALL APPROACH}

A 3D model representation of leaks in WSSs is of great interest. For example, a 3D representation of a laboratory simulated water leak can be observed in Tavera (2008). In this work, Kirchhoff migration and the Hilbert transform were used as pre-processing methods. Although this work is promising, it only presents the leak in a partial manner and based on obtaining the first hyperbolae. In contrast, we assess the feasibility of extracting contours, provide information beyond the first hyperbolae, and enable more robust $3 \mathrm{D}$ representations that show the development of the phenomenon over time.

The most important benefits of 3D representation include a clear visualization of the studied phenomenon; support for feasibility studies of hydraulic behavior in WSSs; and, 
finally, support for technical WSS management - such as maintenance, rehabilitation, and general decision-making.

The difficulties associated with this study are related to the GPR images from which data was extracted. The first drawback was the absence of clear protocols for performing image interpretations when there is excessive noise in the images - such as radio-waves, mobiles, and electrical sources (Brito-Schimmel et al. 2010) - which cause deformation in the response from the buried objects and made the task of identification harder. This means that interpretation becomes more subjective. Consequently, algorithms were developed that enabled automatic 3D leak modeling and so avoided subjectivity.

\section{CASE STUDY: THE LAYOUT FOR THE LAB TESTS}

We describe in this section the layout of the lab tests (Figure 1). For the set of the tests performed, a plastic pipe commonly used in WSSs was buried in dry soil in a tank.

Figure 1. Survey configuration: (a) pipe burying process in the laboratory tank, (b) polypropylene plate - antenna sliding surface, (c) control set schematic configuration.

The characteristics of the buried pipe were: (a) PVC; (b) diameter of $100 \mathrm{~mm}$; (c) length of $0.95 \mathrm{~m}$; (d) hole drilled to simulate the leak in the central part of the pipe; (e) two points for water input and output with connections at the ends (see Figure 1.a). A wooden tank measuring $1.0 \mathrm{~m} \times 1.0 \mathrm{~m} \times 0.60 \mathrm{~m}$ was used. After the pipe was positioned, its supports were removed, and it was then covered with dry soil (before introducing water into the system). The surface of the tank was covered with a polypropylene plate (see Figure 1.b). Eleven paths parallel to the X-axis and another eleven paths parallel to the Y-axis were marked on this plate. These 22 paths were spaced $0.10 \mathrm{~m}$ apart to form the sampling grid. Three of these 22 paths were parallel to the pipe, and one was positioned in a transversal direction. We term this layout the control set (CS); each control set was composed of four slices, $s$, with domain $\{\mathrm{A}, \mathrm{B}, \mathrm{C}, \mathrm{D}\}$. The three parallel slices (sA to $\mathrm{sC}$ ) were $400 \mathrm{~mm}$ long and the transversal slice (sD) was $700 \mathrm{~mm}$ long (see Figure 1.c). The selection of these profiles crossed the pipe in longitudinal (CSsC) and transversal (CSsD) directions, both profiles intersecting azimuthally above the leak point (see Figure 1, c). The other two profiles were placed close to the pipe and intended to keep track of the leak. A GPR antenna was placed on each line of this mesh.

The GPR equipment used in each survey was a commercial monostatic antenna with a central frequency of $1.5 \mathrm{GHz}$. The parameters of the equipment were 120 trace/s, 512 samples/trace and $20 \mathrm{~ns} / 512$ samples.

The total duration of the tests, corresponding to a timeframe of 35 minutes was divided into three stages. Stage 1 starts at $[(t 0,00: 00: 00)]$ with the initial condition so that the test is performed under the established configuration with no water in the system. Stage $2[(\mathrm{t} 1,00: 02: 10),(\mathrm{t} 2,00: 06: 02)]$ involved filling the pipe. Times $\mathrm{t} 1$ and $\mathrm{t} 2$ correspond to a volume of water less than that required to fill the pipe. Stage 3 [(t3, 00:11:42), (t4, 00:14:02), (t5, 00:17:03), (t6, 00:21:54), (t7, 00:28:05), (t8, 00:33:06), (t9, 00:35:06)], in turn, corresponds to the water added to the system once the pipe has been filled. 


\section{INTERPRETATION AND CLASSIFICATION OF THE GPR PROFILES}

The proposed approach to extract contours of the GPR images is presented in this section. The process follows these steps: a) raw image pre-processing; b) pre-processed image abnormality analysis and location; c) interesting abnormality color range identification; d) contour extraction; and e) 3D model representation. This process is described below.

The data obtained from the GPR laboratory surveys was the main input and was visualized as radargrams consisting of the equipment received signals stored in a matrix $\left(M B\right.$, raw image matrix, Figure 2.a). This matrix was composed of $m$-vectors $b_{k}$, $k=1, \ldots, n$ (traces), representing the change in depth of the electromagnetic inspected soil properties. Arranging this matrix by columns, we obtained $M B=\left[b_{1}, b_{2}, \ldots, b_{n-1}, b_{n}\right]$. Vector $b_{k}$ of length $m$ corresponds to the registered data volume in each trace, depending on the characteristics of the equipment (in our case 512 data per trace). Considering the difficulties for raw data analysis caused by the poor visibility of some objects (based on their electromagnetic characteristics), a raw image pre-processing methodology is proposed in this document that helps improve visualization of abnormalities contained in the survey images.

The pre-processing algorithm used was proposed by Ayala-Cabrera et al. (2013a). The algorithm is based on game theory and uses the so-called multi-agent paradigm. Specifically, the process is simulated by a race of agents, as explained below. The algorithm input in our case is the radargram resulting from the GPR prospection, consisting of the $m \times n$ matrix, MB. The GPR prospection generated consists of $n$ traces, which were employed as parallel tracks to be walked by the $n$ agents. The race is an endurance test for the competing agents, the prize for each agent being a movement step for each effort performed. Those efforts are based on wave amplitude value changes in each column of the matrix (Ayala-Cabrera et al. 2013a). The race takes a total time $t t=$ $t w+t r=m, t w$ being the warm up time and $t r$ the competition time. The displacements of the agents during time tr are conditioned by the trend change of the wave amplitude on the trace that is being run. The race ends when time $t t$ has elapsed, and the race winner is the agent who has obtained the largest displacement during this time. Output1 (Figure 2.b) of this process consists of an $m 1 \times n$ matrix, $m 1$ being the maximum number of displacements. Columns in this matrix describe the movement of the agents related to the competition. In this work, the movements obtained by the agents are called time lines. On each time line, the time obtained in the competition by each agent is sorted by increasing values and indexed from 1 - giving equal index to equal times. These time lines are later normalized, obtaining Output2 (Figure 2.c) which is the matrix used in the sequel.

Output2 results are subjected to detailed visual analysis, and compared to detect characteristics that give information regarding the water development in the CS. The zone affected by the water input into the system was identified by comparing the current time of each slice with the previous time, thus observing the appearance of new forms for the current time. In cases in which the only variation in the system is the input of water from the leak, the presence of a new form in the evaluated slices was associated with the trace left by the water input. Once the affected zone is identified in each image 
of Output2, an iterative segmentation is made, based on one or more ranges ([min max]. Figure 2.d) contains the identified zone as the zone affected by the water input. As a result of this range selection, a matrix (Output3) is obtained that has the same dimensions as Output2: if $v \geq \max$ and $v \leq \min$, then $v 1=1$, otherwise $v 1=0\}$, where $v$ is the data value in Output2, and $v 1$ is its corresponding binary value in Output 3.

From Output3 a contour detection was performed (Figure 2.e) using the MooreNeighbor tracing algorithm modified by Jacob's stopping criteria (González et al. 2004), implemented in Matlab's bwboundaries function. From those generated contours a selection that shows spatial continuity when compared with previous time step contours was made. The selected contours were transferred to the original raw image space. Finally, to improve comprehension and visualization of the results, a fusion of the different contours (in the original space) was performed for each capture set. Fusion of the shapes obtained from GPR images after the iterative interpretation was performed using 3D models. This fusion was performed by positioning the points at their respective spatial coordinates and then plotting the end points for each group. These extreme points were connected by a Delaunay triangulation, which enabled surface visualization.

The contours obtained in this process will serve in future research to train intelligent data classification systems that can automatically detect contours. This can be achieved by using shape characteristics (such as eccentricity and orientation) and system parameters (such as pressure, water volume, and soil humidity) and the final objective is to validate or generate models that facilitate an improved understanding of the phenomenon.

The aim is to improve the visualization in subsequent extraction and classification of contours.

Figure 2. An example of the applied process: (a) raw image - $M B$ matrix; (b) preprocessed image - Output1; (c) normalized pre-processed image - Output2; (d) image after range selection - Output3; (e) contouring, group selection; and (f) contour extraction and retrieval.

\section{ANALYSIS OF RESULTS}

In this section, the obtained results are presented after the GPR image interpretation and classification process. It must be mentioned that although the selected contours do not cover the total leakage area, a considerable part of this area is effectively selected. This means that the obtained results show some deformation and are not fully accurate. Nevertheless, these results give relevant information about the represented phenomenon. We also claim that, with some improvements, complete information could be automatically extracted. The obtained results of the process of interpretation and extraction of contours are shown in Figure 3. 
Figure 3. Leakage area contour slices A through D

Figure 3 shows that times corresponding to Stage 2 ( $t 1$ and $t 2)$ in the slices from A through $\mathrm{C}$ do not present a significant difference, which is coherent with the survey plan when considering that this is the pipe filling stage. From Stage 3 ( $\mathrm{t} 3$ through $\mathrm{t} 9$ ), the pipe has reached its maximum volume and starts leaking; it is just after $\mathrm{t} 3$ that the slices start to demark the zone being filled with leaked water. It is observed that from $\mathrm{t} 3$, in slices $\mathrm{A}$ and $\mathrm{B}$, the extracted contours present a similar form. For slice $\mathrm{C}$ the first demarcating contour appears at $t 4$. The difference between the extracted contours lies in the difference in zone growth and in ascending contour displacement, caused by the volume increase and rising water.

In slice $\mathrm{D}$, the results corresponding to the transversal slice for the pipe over the leak spot are shown. For Stage 2, in $\mathrm{t} 1$ and $\mathrm{t} 2$, it is observed that it is possible to extract the pipe zone in the filling process, in contrast to the previous slices (slices A through $\mathrm{C}$ ). The pipe is demarked by a hyperbola and this is observed in all the images; the contours are fully ( $\mathrm{t} 1$ and $\mathrm{t} 2$ ) or partially ( $\mathrm{t} 3$ through $\mathrm{t}$ ) ) enhancing the pipe (zone within the hyperbola) and leaked water (remaining zone). In Stage 3, the selected zone set grows and takes the main role in the image, enabling the water action to be seen once it is out of the pipe.

The variation of the forms in the various images shows that it is possible to obtain (to some extent) the path of the water as it leaks from the pipe. While the results are not accurate and reveal some deformation, it is also true that they can be calibrated to establish a model of leaked water for each type of breakage, and relate the leak with the pressure and flow values in the system. Similarly, a more complete study with different soil types could serve to identify the type of propagation and model the plume dispersion.

With the objective of better understanding the presented phenomenon in Figure 3, Figure 4 shows the results obtained in 3D representations to improve visualization.

Figure 4. 3D model leak evolution; (a) to (i) represent times t1 to t9, respectively.

Figure 4 shows the volume form caused by the leaked water through the different survey stages, making it evident the difference between Stages 2 and 3 . The corresponding volumes show the evolution of the leak over time in the area of interest. Form and volume variation is observed as the leakage grows, along with its development. Additionally, it is possible to appreciate how the water leak tends to grow upwards. This enables determining the tank volume that is affected by the simulated water leak, thus generating the base to create theoretical models of dispersion under less controlled conditions. The 3D images obtained by using this approach may enable personnel who are not non-highly qualified in GPR to understand the phenomenon of a buried water leak. 


\section{CONCLUSIONS}

From this study we can conclude that it is feasible to use nondestructive methods (GPR in our case) in water leak detection from buried pipes to rebuild the stages of the leak and show how water is dissipated underground. Since this work shows that it is possible to obtain the wet area contours, from which more complex studies can be made, it is also possible to determine the volume of wet soil, and volume change over time. From this perspective, we conclude that the obtained contours of this study contribute information beyond the initial hyperbolae study that some previous works rely on. This approach favors the generation of more accurate models in terms of reproduction or detection of leaks.

In addition, the application of a pre-process over the GPR images enables the image data to reveal more information based exclusively on this data (that is to say, with no reference or contrast images) and so improves visualization. This brings us closer to an automatic detection and location of these abnormalities. The work performed has shown the feasibility of generating 3D models that are easily interpreted by personnel who are not highly qualified in the use of GPR.

\section{Acknowledgements}

Part of this work has been developed under the support of an FPI (Formación de Personal Investigador)-UPV (Universitat Politècnica de València) scholarship granted to the second author by the Programa de Ayudas de Investigación y Desarrollo (PAID) of the Universitat Politècnica de València, and the support of Fundación Carolina PhD, within its short stances scholarship program for the first author. The use of English in this paper has been revised by John Rawlins.

\section{REFERENCES}

